\title{
An experimental study of exploiting multipath fading for robot communications
}

\author{
Magnus Lindhé and Karl Henrik Johansson \\ School of Electrical Engineering \\ Royal Institute of Technology \\ Stockholm, Sweden \\ \{lindhe | kallej\}@ee.kth.se
}

\author{
Antonio Bicchi \\ Interdepartmental Research \\ Center "Enrico Piaggio" \\ University of Pisa, Italy \\ bicchieing.unipi.it
}

\begin{abstract}
A simple approach for mobile robots to exploit multipath fading in order to improve received radio signal strength (RSS), is presented. The strategy is to sample the RSS at discrete points, without deviating too far from the desired position. We first solve the problem of how many samples are needed for given communications performance and how they should be spaced. Second, we propose a circular and a grid trajectory for sampling and give lower bounds on how many samples they will yield. Third, we estimate the parameters of our strategy from measurements. Finally we demonstrate the validity of our analysis through experiments.
\end{abstract}

\section{INTRODUCTION}

In many applications for multi-agent robotics, such as surveillance, mapping of unknown environments and searching, the result is of no value unless it can be communicated to a base station or operator. A need arises to ensure that robots perform their tasks in a "communications aware" manner, to avoid losing contact with their team-members or the outside world. There are several approaches to doing this, for example by restricting the movement to preserve connectivity [8], preserving a clear line of sight [1] or deploying relaying agents if the link is getting too weak $[15,14,10]$. A similar idea is to use mobile agents to bridge disconnected groups of networked sensors [2]. Most of the proposed coordination schemes have been developed based on assumptions of very simplified channel models. A popular approach is to use a binary link model where agents can communicate perfectly within a certain radius, but not at all outside it [3]. Slightly more sophisticated approaches assume that the signal strength decays according only to the path loss $[11,9,6]$. In indoor environments, this overlooks the very significant multipath fading effect that has been extensively studied in the field of electromagnetic wave propagation. In this paper, we show that awareness of this enables us to make significant improvements in the achieved communication performance for multi-robot systems. There are several other ways of counteracting the multipath fading, such as antenna diversity, adaptive antenna arrays or frequency spreading. But antenna diversity or arrays require a certain physical size of the robot and so may not be feasible for very small platforms. Frequency spreading, on the other hand, is not always very effective, as shown in our measurements and by Puccinelli et al. [12].
The contribution of this paper is to formulate the problem of positioning the robot to alleviate the effects of multipath fading, and to validate the proposed approach against real data. We consider static environments, where only the robot is moving so that the fading does not vary over time. Such scenarios are relevant in contexts of, e.g., robotic search and surveillance. The problem is divided into two parts: first we provide an estimate of the number of points that the robot needs to sample to find a position that has a given signal strength. Then we suggest two possible sampling strategies to collect the required number of independent samples, without deviating too far from the desired path of the robot. It is shown that the approach is robust and works in several kinds of propagation environments, and almost certainly avoids the deep fades where the signal can be attenuated as much as 20 $\mathrm{dB}$. In most cases, the strategy gives a gain of $5 \mathrm{~dB}$ or more compared to the local mean signal power. A demonstration in a simple experiment with a single robot is provided.

This paper is organized as follows: In Section II, we develop our channel model and explain the phenomenon of multipath fading. We then formally state the problem in Section III and solve it in the case of perfect Rayleigh fading in Section IV. The assumptions for Rayleigh fading are not, in general, true in actual environments, so in Section V we present measurements to estimate the statistical properties of fading in representative environments. Finally we present experimental validation of our approach on a robot platform in Section VI, and end with some conclusions in Section VII.

\section{COMmUnicATIONS PRELIMINARIES}

In this section we review some results on what determines the received signal strength (RSS) in a radio receiver and how this is affected by the surrounding environment. Unless stated separately, this section follows the book by Stüber [13].

Given a transmission power of $P_{t}$, and a distance $d \mathrm{~m}$ to the receiver, the nominal signal power $P_{r}$ in the receiver is

$$
P_{r}(d)=P_{t}+G_{t}-P L_{0}-10 n \log _{10} d+G_{r}[\mathrm{~dB}] .
$$

Here $P L_{0}$ is the path loss at $1 \mathrm{~m}$ from the antenna and $n$ is the path loss exponent. $G_{t}$ and $G_{r}$ are antenna gains in the transmitter and receiver, respectively. The path loss exponent $n$ is 2 for free-space propagation and can reach as high as 4 in 


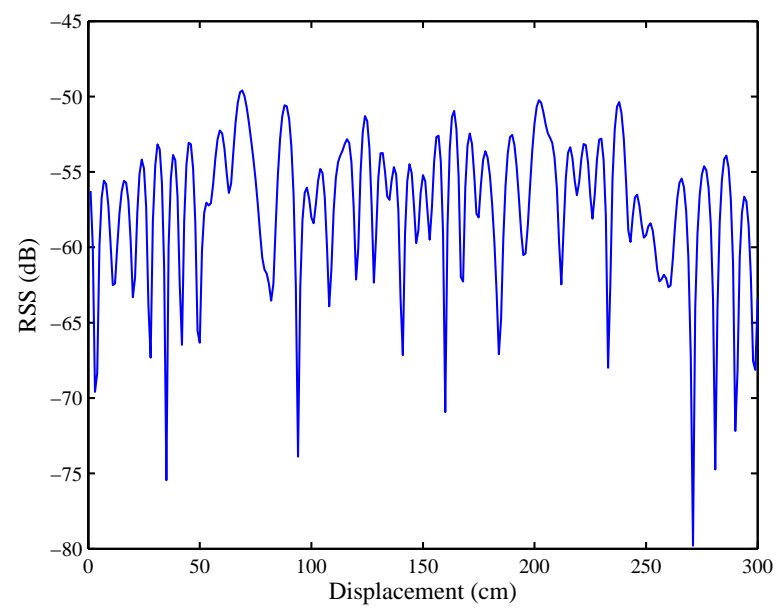

Fig. 1. Simulated Rayleigh fading at $2.4 \mathrm{GHz}$ as the receiver moves along a $3 \mathrm{~m}$ line, taking samples of the RSS at every $\mathrm{cm}$.

some environments. At $2.4 \mathrm{GHz}$ and in an office environment, values around 2.5 have been reported [5].

In an urban or indoor environment, there are however large fluctuations around the nominal level due to shadowing and multipath fading. Shadowing is caused by objects obstructing the signal path and varies over distances the same order as the obstructions.

Multipath fading, on the other hand, is caused by destructive or constructive interference between the signal and its reflections and it varies over very short distances, in the order of a wavelength. Even a stationary receiver will experience multipath fading if the environment is changing, for example due to cars and people moving or doors opening and closing. If all signal components that reach the receiver are of equal strength, the multipath fading is called Rayleigh fading, while if there is a line-of-sight (LoS) component that is significantly stronger, we have Ricean fading.

It should be pointed out that for given antenna positions, the fading is reciprocal and thus affects the signal path equally in both directions. But its spatial properties in general are not. Specifically, if a base station with very open surroundings is communicating with a robot in a cluttered environment, the multipath fading varies over much longer distances at the base station than at the robot.

Due to the difficulty of predicting the Rayleigh fading, it is usually modeled as a stochastic effect. The probability density function (pdf) of the RSS in a Rayleigh fading environment is

$$
f_{P}(x)=\frac{1}{P_{r}} \exp \left(\frac{-x}{P_{r}}\right) .
$$

The expected value is $P_{r}$.

A simulated Rayleigh fading signal power plot is depicted in Figure 1. The spatial autocorrelation of the fading envelope as a function of the distance $\delta$ between two samples is

$$
R(\delta)=k J_{0}^{2}\left(2 \pi \delta / \lambda_{c}\right)
$$

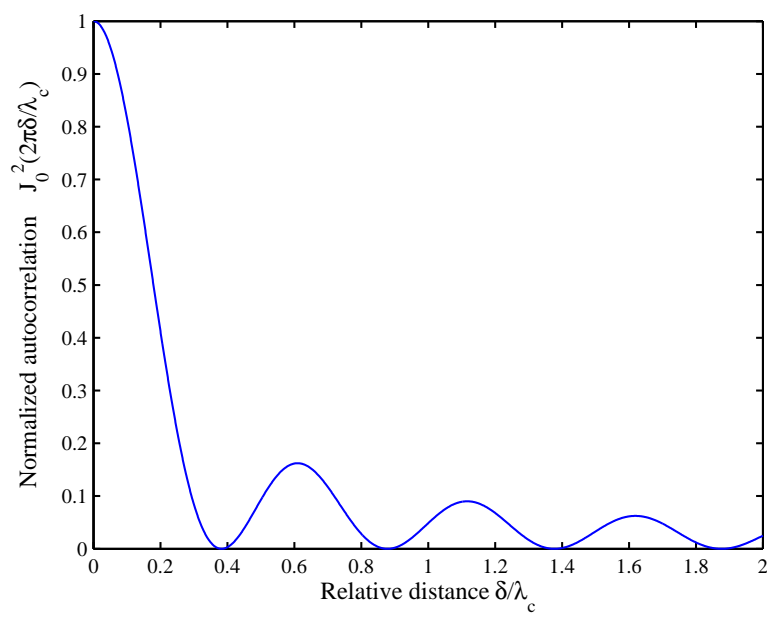

Fig. 2. Normalized spatial autocorrelation of the RSS in a Rayleigh fading environment.

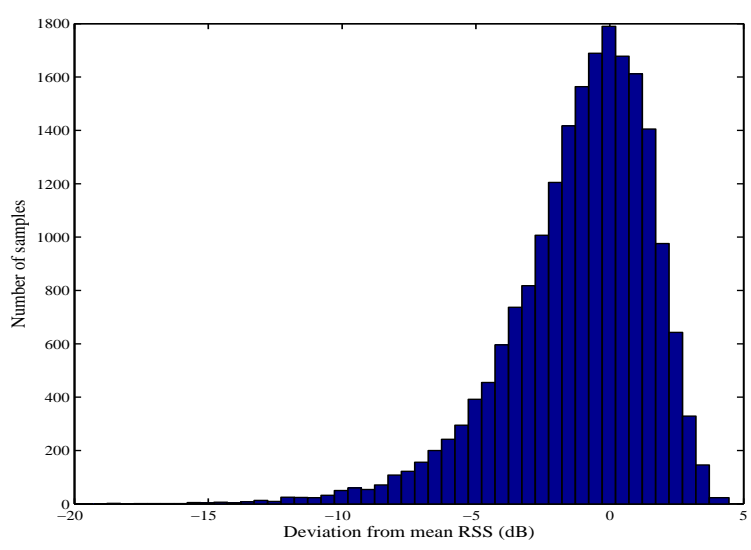

Fig. 3. A histogram based on 20000 simulated measurements, showing the typical Rayleigh distribution.

where $k$ is a constant, $J_{0}$ is the zero-order Bessel function of the first kind and $\lambda_{c}$ is the carrier wavelength. This is illustrated in Figure 2. It shows that two samples taken $0.38 \lambda_{c}$ apart $(4.75 \mathrm{~cm}$ at $2.4 \mathrm{GHz})$ should have zero correlation, and samples taken at a greater distance should always have small correlation. In practice, samples taken more than about half a wavelength apart are considered to have independent Rayleigh fading. For comparison with measurements in later sections, we also include a histogram of 20000 simulated samples of Rayleigh fading in Figure 3. The histogram is normalized by subtracting the mean (computed in $\mathrm{dB}$ ) to allow comparison with the measurement results in later sections.

\section{PROBLEM FORMULATION}

In this section we formulate the problem of coordinating a robot to improve its communication capability by counteracting multipath fading. We consider situations when the application allows the robot to deviate slightly from the desired 


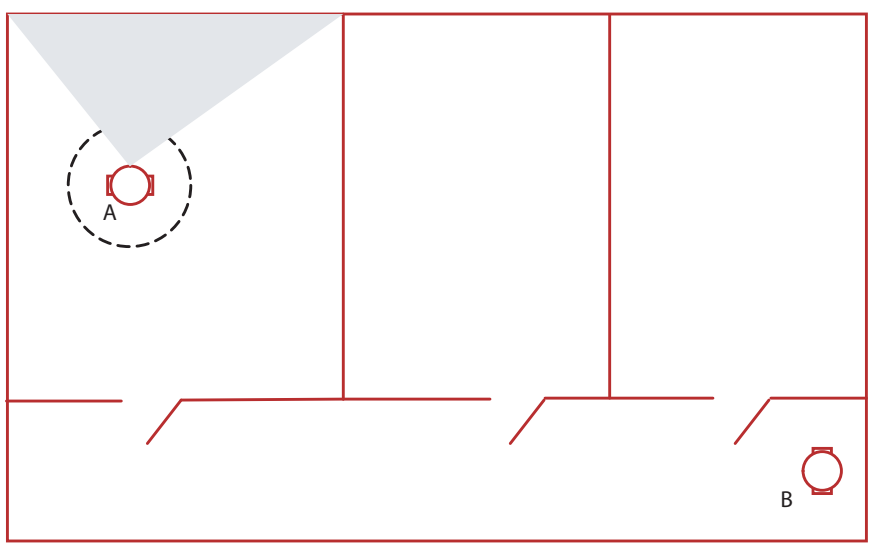

Fig. 4. A scenario where a robot (A) enters a room to monitor part of it, and send the data back to another robot (B). The task can be fulfilled from anywhere inside the dashed circle, so the robot can move inside it to improve the communications with B.

position, in order to improve the communication. A typical scenario is demonstrated in Figure 4, where robot A monitors a room and needs to send data to robot $B$ in the corridor outside. The task allows A to deviate within a given region in search of higher signal strength.

Since the RSS is practically impossible to predict and has multiple local maxima, finding the optimal position would require visiting all of the feasible region. We therefore suggest sampling the RSS in a finite number of points, and then going back to the best. This requires high-accuracy navigation, which is not always available. An alternative is to sample a few points to estimate the nominal RSS and then continue the sampling, stopping at a point that offers a given improvement over the nominal level. We can then statistically express what improvement of the RSS this will give. Our first problem is thus formulated as:

Problem A: Find the number $N$ of independent samples that we need to get an improvement of $G \mathrm{~dB}$ over the nominal RSS, with probability $P$.

The trajectory should allow for simple control laws and not deviate outside the feasible region. We have concentrated on what we believe to be the most common robot types today car-like robots or robots with differential drive. The kinematics of a differential drive robot are

$$
\begin{aligned}
\dot{x} & =v \cos \theta \\
\dot{y} & =v \sin \theta \\
\dot{\theta} & =u,
\end{aligned}
$$

where the robot has position $(x, y)$ and bearing $\theta$, and the control inputs are the angular velocity $u$ and linear velocity $v$. For car-like robots,

$$
u=\frac{v}{L} \tan \alpha,
$$

with $L$ being the distance between rear and front axels, and $\alpha$ the steering angle, limited by $|\alpha| \leq \alpha_{\max }$.

Problem B: Find a trajectory that is simple to follow with a car-like or differential drive robot, and offers $N$ sampling points, spaced at least $\Delta$, without deviating more than $R$ from the original position.

As a remark, applications such as transportation or patrolling may require the robot to follow a trajectory. In this case the robot may sample the RSS along its trajectory, and stop to communicate when it finds a good position. Problem $A$ is then of interest to give an upper bound on the number of samples (thus also the time or distance) between successful communication attempts.

The general problem has thus been divided into two parts: First finding the number of samples $N$ required to achieve the desired performance and, second, finding a suitable trajectory for the robot to visit that many sampling points. In the following, we provide solutions first in the theoretical case of Rayleigh fading, and then based on properties of real environments.

\section{SOLUTION IN PERFECT RAYLEIGH FADING ENVIRONMENT}

In this section we give a solution with provable properties in the case of perfect Rayleigh fading. We first give a proposition on the number of samples required to achieve a certain gain and then suggest two alternative strategies of fitting the required number of independent samples within the region where the robot is allowed to move.

Proposition 4.1 (Number of samples): For a Rayleigh fading environment, the number of independent samples $N$ needed to achieve a power gain of $G \mathrm{~dB}$ with probability $P$ compared to the nominal RSS is given by

$$
N=\frac{\ln (1-P)}{\ln \left(1-\exp \left(-10^{G / 10}\right)\right)} .
$$

Proof: From Equation 1, we have the pdf of the signal power, which gives the cumulative distribution function (cdf)

$$
C\left(P_{n}\right):=\operatorname{Prob}\left(X<P_{n}\right)=1-e^{-P_{n} / P_{r}}
$$

i.e., the probability that the power in a single sample is lower than the threshold $P_{n}$. Taking $N$ independent samples, the probability that all of them are lower than $P_{n}$ is $C\left(P_{n}\right)^{N}$. We note that at least one sample being greater than $P_{n}$ is the complementary event to the above, and since $P_{n} / P_{r}=10^{G / 10}$, the probability of this is

$$
\operatorname{Prob}(G)=1-\left[1-\exp \left(-10^{G / 10}\right)\right]^{N} .
$$

Solving for $N$ gives the proposition.

As described in Section II, samples taken at a distance of $0.38 \lambda_{c}$ can be considered independent. This can be viewed as each sample being surrounded by a disc of radius $0.19 \lambda_{c}$ where no more samples should be taken. So taking $N$ independent samples inside the feasible region with radius $R$ is essentially a two-dimensional sphere-packing problem.

We propose two possible sampling trajectories; driving in a circle and sweeping a hexagonal lattice. They represent different trade-offs between ease of navigation and maximizing the number of samples. 

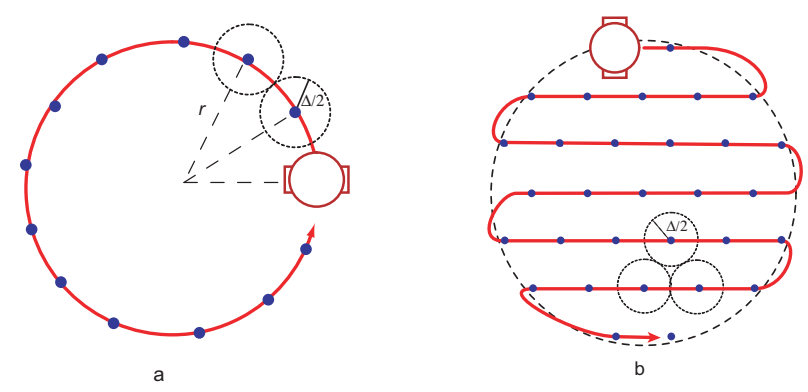

Fig. 5. Two possible sampling trajectories: a circle (a) and a hexagonal lattice (b). In both cases, the distance between sampling points is at least $\Delta$.

Proposition 4.2 (Circular sampling trajectory): If $N$ samples are taken on a circle, and the samples are at a distance not less than $\Delta$, the radius of the circle must be

$$
r \geq \frac{\Delta}{\sqrt{2} \sqrt{1-\cos (2 \pi / N)}}
$$

This is illustrated in Figure 5a. Another possible sampling pattern is the intersection of a hexagonal lattice and a circle with radius $r$. A hexagonal lattice can be defined as

$$
\left\{(x, y)=\Delta(k+a, \ell \sqrt{3} / 2): a=\frac{1}{2} \bmod (\ell, 2), k, \ell \in \mathbb{Z}\right\}
$$

which was proven by Thue [7] to be optimal for two dimensional sphere-packing. The distance $\Delta$ is the vertex distance. This arrangement of sampling points is also suitable for being covered by differential drive or car-like robots and with collision sensors also in the back, one could reverse along every second line to simplify maneuvering. Sensors such as a camera can be pointed in the interesting direction during the whole sampling procedure. If the robot detects an obstacle, it can simply turn earlier and start following the next line back. A hexagonal lattice with sampling trajectory is depicted in Figure $5 \mathrm{~b}$. The required size of the sampling region is stated by the following proposition:

Proposition 4.3 (Hexagonal lattice of samples): A hexagonal lattice with vertex distance $\Delta$ has at least $N$ vertices within the distance

$$
r=\left[\sqrt{\frac{\sqrt{3}(N+1)}{2 \pi}}+\frac{1}{\sqrt{3}}\right] \Delta
$$

from the origin.

Proof: Each vertex can be regarded as the center of a hexagon with area $\sqrt{3} \Delta^{2} / 2$, as shown in Figure 6a. A circle of radius $a$ has an area equal to or greater than the area covered by

$$
N=\left\lfloor\frac{2 \pi a^{2}}{\sqrt{3} \Delta^{2}}\right\rfloor
$$

such hexagons. The hexagons can be tiled so that their centers all fit within a circle of radius $a+\Delta \sqrt{3}$, see Figure $6 \mathrm{~b}$. This can be proved as follows.

Assume that any hexagon is completely outside the circle. Since the remaining hexagons cannot fill the circle, there must
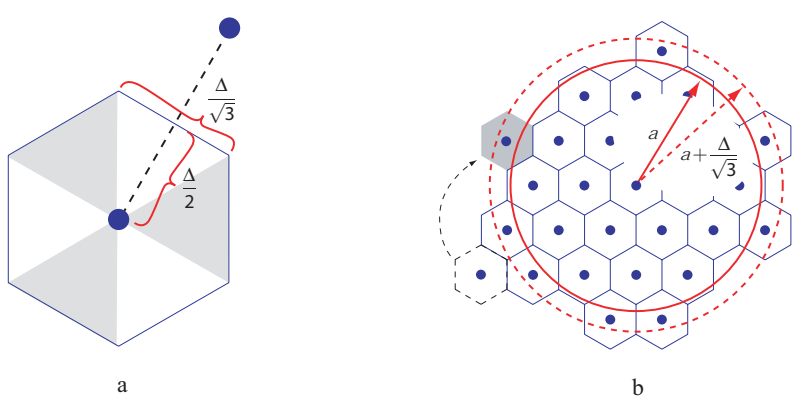

Fig. 6. (a) The sampling pattern follows a hexagonal lattice. (b) If one of the $N$ hexagons (dashed) is completely outside the circle of radius $a$, there must exist a free space partially inside the circle, where it can be moved (in gray).

be some free space partially inside, and since hexagons can be tiled with no gaps, this space must be on the perimeter. So the hexagon can be moved there instead. To complete the proof, we also note that no part of a hexagon is more than $\Delta / \sqrt{3}$ from its center, so since all hexagons have some part inside the circle of radius $a$, their centers must then fit inside a concentric circle of radius $a+\Delta / \sqrt{3}$.

Solving Equation 6 for $a$, using that $N+1 \geq\lfloor N\rfloor$ and adding the margin $\Delta / \sqrt{3}$, gives the proposition.

Other trajectories than the two described here are of course also possible; the fewer samples needed, the greater the flexibility to choose a trajectory.

\section{MEASUREMENTS IN REAL ENVIRONMENTS}

To apply the proposed strategy in a real environment, we need to estimate the spatial correlation (to determine the sample spacing) and the cdf of the signal strength. We have chosen a basement corridor and a cluttered lab room as representative environments for the measurements.

To automate the measurements, we have mounted a radio based on the CC2420 chip on a robot. It communicates with the same chip on a TMote Sky wireless sensor node, connected to a PC, see Figure 7. The CC2420 operates at $2.4 \mathrm{GHz}$ with a maximal output power of $0 \mathrm{dBm}$ and has a softwareaccessible received signal strength indicator (RSSI) [4]. It is worth noting that the CC2420 uses direct sequence spread spectrum modulation. This is supposed to alleviate the effects of multipath fading, but as shown below and in [12], the problem of deep fades remains.

The TMote has an antenna integrated on its circuit board, while the robot has a quarter-wave antenna on top. The integrated antenna is by design not omnidirectional, and measurements show that the antenna mounted on the robot also has some directional dependence, due to the influence from the rest of the robot. This makes it important that, when the robot has found the best position and returns there, it also returns to the same orientation.

To estimate the spatial correlation in the different environments, we have driven the robot along straight $200 \mathrm{~cm}$ lines, stopping and sampling the RSSI each centimeter. Each sample 


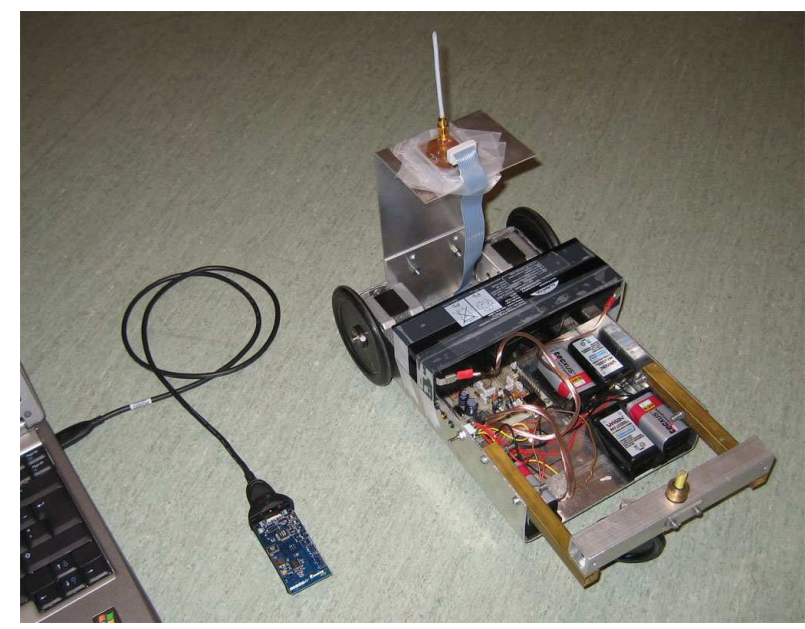

Fig. 7. The measurement system, with the robot and the TMote connected to a PC. The robot has two driving wheels and a third caster wheel, and its antenna is positioned about $25 \mathrm{~cm}$ above ground.

is formed from averaging the RSSI readings from four radio packets. For each sequence of $N=200$ samples, we computed the unbiased estimate of the autocorrelation

$$
\hat{R}(k)=\frac{1}{N-|k|} \sum_{m=0}^{N-k-1}[z(m)-\bar{z}][z(m+k)-\bar{z}]
$$

where $z(n)$ is the signal envelope in sample $n$ and $\bar{z}$ is the mean value.

The cdf of the RSS samples was estimated using the same measurement setup, but driving a distance greater than $\Delta$ between samples. We then assumed that the samples could be regarded as independent. Since the nominal RSS is difficult to calculate, we estimate it by the local average. The result is plotted in a histogram. Summing over this histogram gives an estimate of the cdf.

The lab room contains lots of computers, metal cabinets and other effective scatterers, so it is our belief that this environment produces near-Rayleigh fading. This is also confirmed by the measurements. One representative measurement series is depicted in Figure 8, and the estimated autocorrelations for five measurements are superimposed in Figure 9. The autocorrelation decays rapidly, and reaches the noise floor at $\Delta=6 \mathrm{~cm}$ in all measurements in this environment. (This matches the predicted $\lambda_{c} / 2=6.25 \mathrm{~cm}$.) Finally, the histogram in Figure 10 indicates that the distribution is very similar to the Rayleigh distribution. The samples were taken with a distance of $10 \mathrm{~cm}$ to ensure independence.

The corridor has metal grates and cable ducts along its sides, and large metal cabinets at the entrance. This means that the radio signal may be efficiently reflected from a few large surfaces, so the robot does not receive signals of equal strength from all directions as required for Rayleigh fading. As shown by the measurements, this gives somewhat different spatial properties to the fading. The RSS fluctuates as in the lab room, but also over longer distances, much like shadowing.

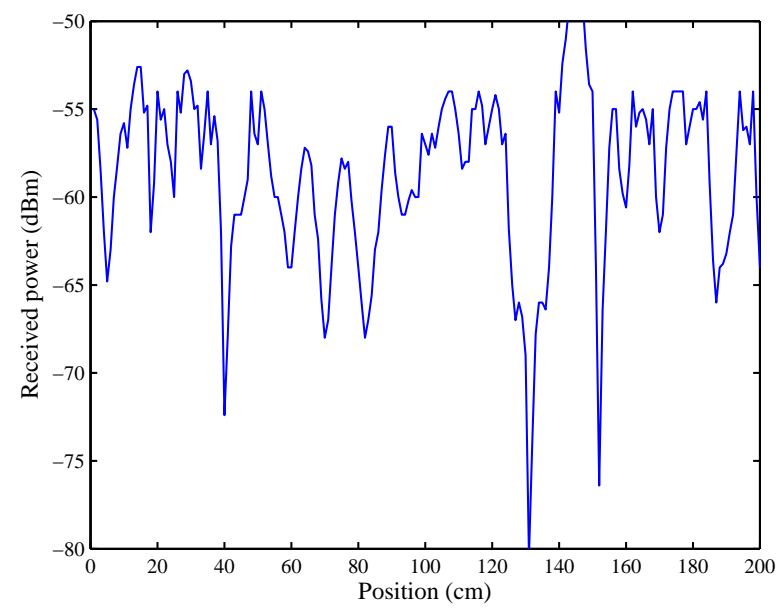

Fig. 8. Measurement results from the lab room, where the RSS varies over $30 \mathrm{~dB}$. Note the deep fade at $130 \mathrm{~cm}$, where the connection was temporarily lost.

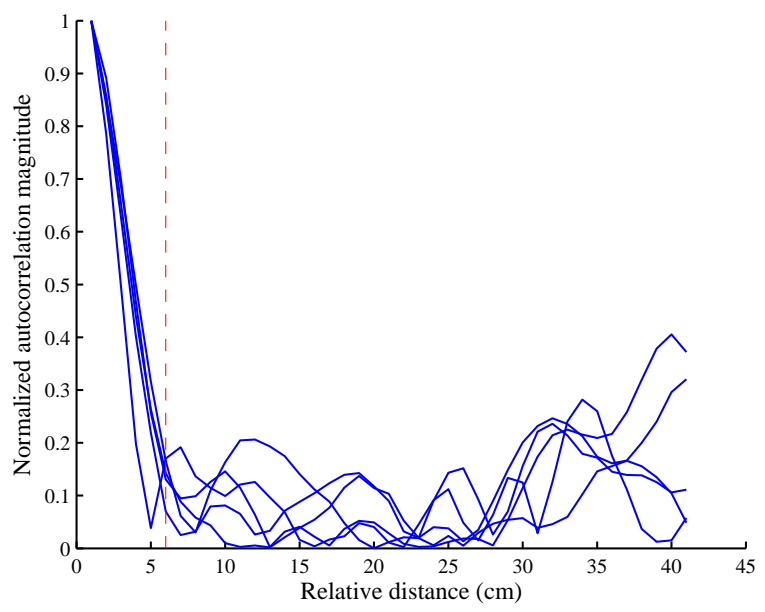

Fig. 9. Autocorrelation estimates for five measurement series in the lab room. The dashed line is the estimated decorrelation distance $\Delta$. The autocorrelation drops rapidly and the spuriouses at $40 \mathrm{~cm}$ are probably due to the estimate being noise sensitive at high lags.

A representative measurement result is illustrated in Figure 11, and autocorrelation estimates for eight measurements are superimposed in Figure 13. The measurement in Figure 11 corresponds to the slowest decaying autocorrelation estimate. At $\Delta=15 \mathrm{~cm}$, all autocorrelation estimates seem to have reached the noise floor for this environment.

To estimate the cdf for the corridor, we took samples 15 $\mathrm{cm}$ apart and collected them in the histogram in Figure 13. Despite the difference in spatial properties, this distribution also resembles that of Rayleigh fading.

The CC2420 data sheet states an RSSI accuracy of \pm 6 $\mathrm{dB}$ and linearity within $\pm 3 \mathrm{~dB}$ [4]. Since we are not interested in the absolute signal power, we therefore consider the measurements to have an uncertainty of $3 \mathrm{~dB}$. During our measurements in static environments, the typical standard 


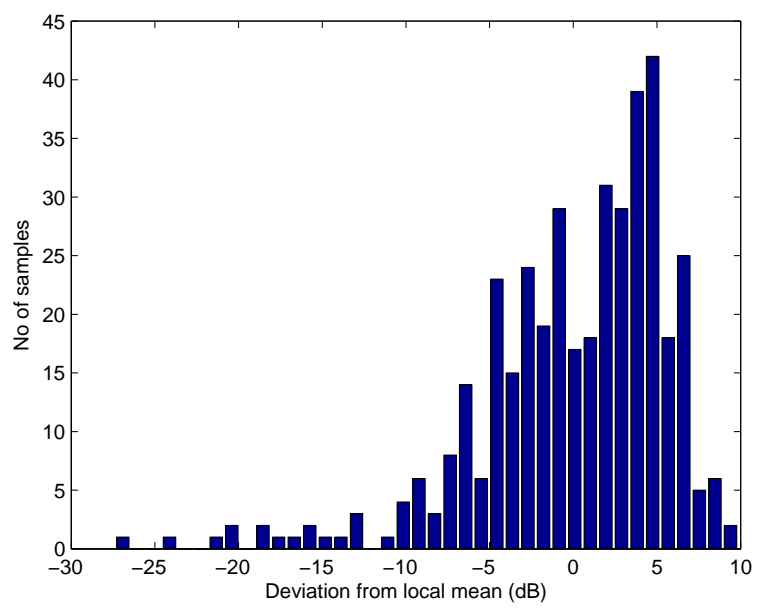

Fig. 10. Histogram of 400 RSS samples, taken in the lab room with sample spacing $10 \mathrm{~cm}$. The distribution resembles the Rayleigh distribution.

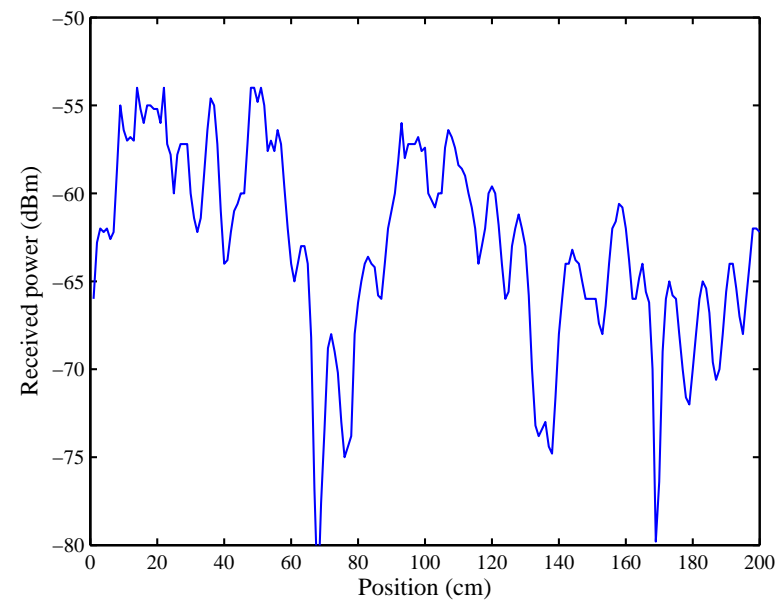

Fig. 11. Measurement results from the corridor. The multipath fading is probably superimposed on a shadowing effect.

deviation within 20 packets was less than $1 \mathrm{~dB}$.

Since our motivating application is autonomous exploration or surveillance of indoor environments, we expect those environments to be static, i.e. with no humans present and little or no movement except for that of the robots themselves. Therefore, the fast fading should not change over time, but only as a function of the position of the transmitter and receiver. To verify this, we made two measurements, first driving the robot forward $100 \mathrm{~cm}$ and then back again along the same line. As illustrated in Figure 14, the RSS as a function of position is very similar between the measurements. The RMS deviation between the measurements is $1.2 \mathrm{~dB}$, i.e., well within the expected uncertainty.

Using the above measurements, we can compute the estimated cdf $\hat{C}\left(P_{n}\right)$. This yields a result similar to Proposition 4.1, but where the signal gain is expressed in relation to the local

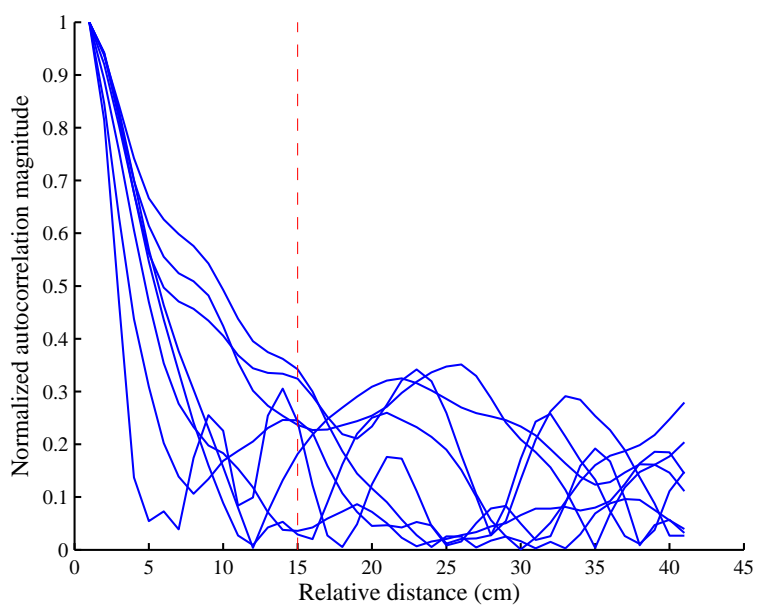

Fig. 12. Autocorrelation estimates for eight measurement series in the corridor. The dashed line is the estimated decorrelation distance $\Delta$. The autocorrelation decays slowly for some series (cf. Figure 9), probably due to shadowing effects.

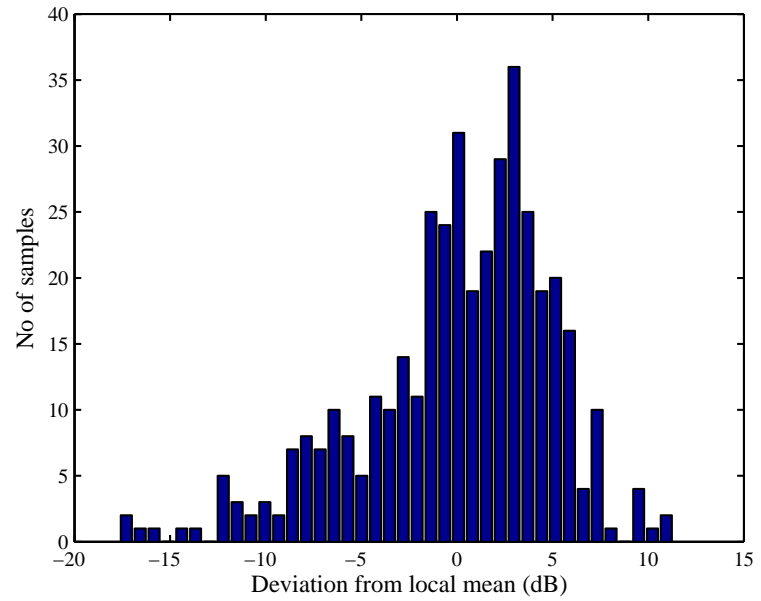

Fig. 13. Histogram of 400 RSS samples, taken in the corridor with sample spacing $15 \mathrm{~cm}$. The distribution resembles the Rayleigh distribution.

average: The probability of achieving gain $G$ when taking $N$ samples can be estimated as

$$
\operatorname{Prob}(G, N)=1-\hat{C}(G)^{N} .
$$

Several curves of $\operatorname{Prob}(G, N)$, for some values of $G$, are plotted for the lab environment as well as the corridor, in Figures 15 and 16, respectively. These figures summarize the answer to Problem A, showing how many samples are needed to reach a given gain with a specified probability.

In practice this means that if the robot can take approximately 9 samples in the lab room (or 14 in the corridor), it has a $95 \%$ chance of finding a position where the signal strength is $5 \mathrm{~dB}$ better than the local average. Under the same conditions, the probability of finding a point where the signal strength is 


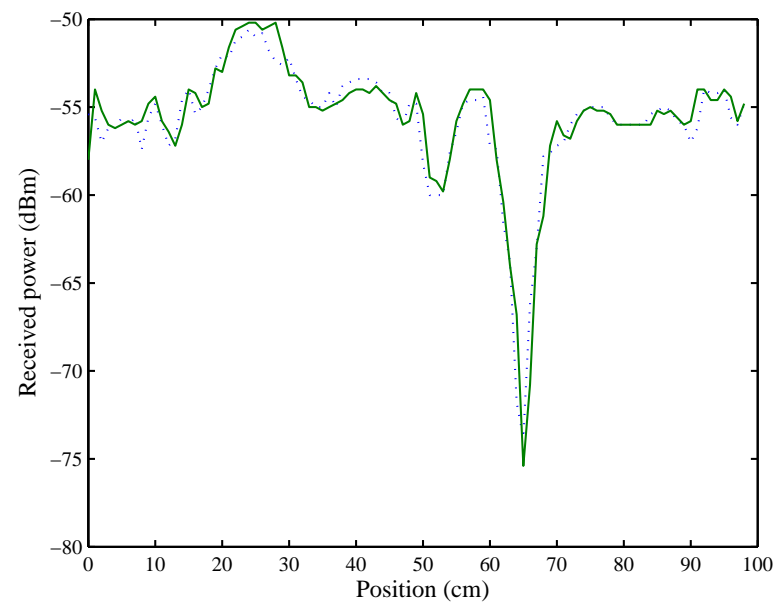

Fig. 14. Two measurement series along the same trajectory. The RSS is clearly a function of the position, and does not vary over time. The RMS deviation between the measurements is $1.2 \mathrm{~dB}$, i.e., within the measurement accuracy.

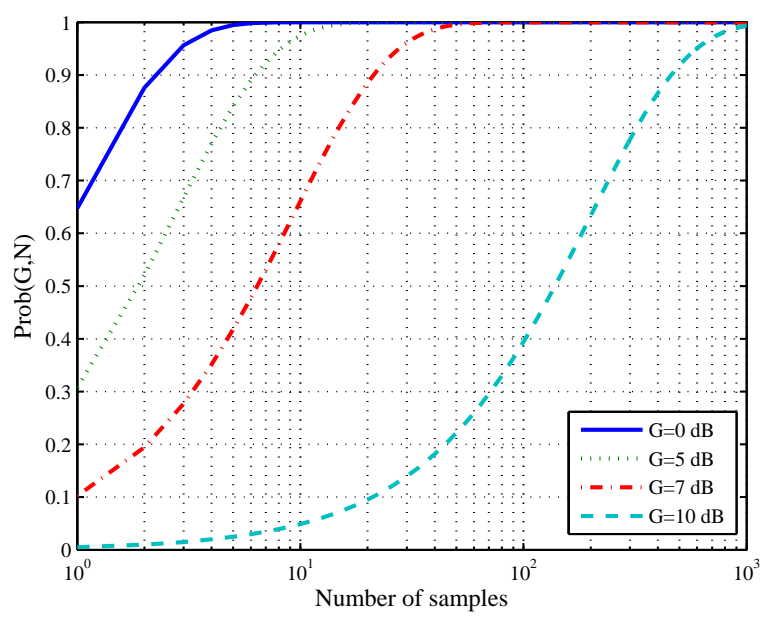

Fig. 15. Results for the lab room: The required number of independent samples of the RSS to achieve a given gain (compared to the local average) with a certain confidence level. We have plotted curves for several gains in the interval $0-10 \mathrm{~dB}$.

at least equal to the local average (and thus avoiding any of the deep fades) is greater than $99.99 \%$. Taking 9 samples in the lab room can be done by going in a circle of radius 8 $\mathrm{cm}$. Conversely, the curves can be used as a guideline for an application designer, choosing the allowed deviation based on what signal gain is needed.

\section{EXPERIMENTAL VERIFICATION}

As a simple demonstration of our suggested approach, we have made experiments positioning the robot at random positions and orientations within a 1-by-1 m square in the lab room, as if a task such as patrolling or mapping had made it drive there. We then measured the signal strength between the

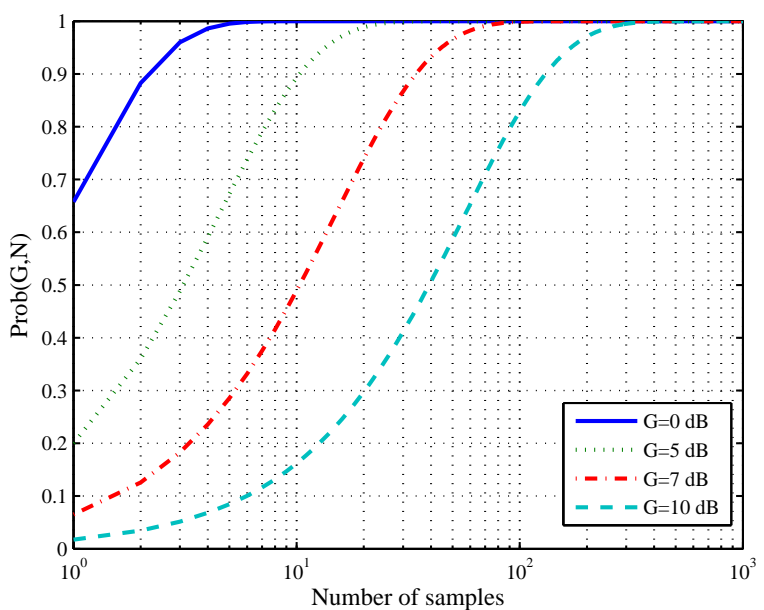

Fig. 16. Results for the corridor: The required number of independent samples of the RSS to achieve a given gain (compared to the local average) with a certain confidence level. We have plotted curves for several gains in the interval $0-10 \mathrm{~dB}$.

robot and its base station (the TMote), in the other end of the room.

First we performed 20 such trials, series 1, allowing the robot to deviate slightly from the initial position, sampling the RSS in 9 points, separated by $5 \mathrm{~cm}$. It then moved to the point with the best RSS before the measurement was made. Then we performed 20 more trials, series 2, where the robot was not allowed to deviate. The result of the two experiment series is plotted as two histograms in Figure 17. When just staying at the initial position, seven out of twenty trials yielded signal strengths worse than the local average, in one case by as much as $15 \mathrm{~dB}$. The theoretical analysis predicted a gain of at least $5 \mathrm{~dB}$ compared to the local average in $95 \%$ of the cases, but in practice this happened in $80 \%$ of the trials. It is worth noticing, however, that all trials avoided negative gains as predicted.

To illustrate the benefit of gaining $5 \mathrm{~dB}$, we quote an expression for the packet reception rate (PRR) as function of the signal-to-noise ratio (SNR), derived by Zuniga et al. [16]. They use MICA2 motes (which work at lower frequencies but have similar performance as the TMotes) with a data rate of $19.2 \mathrm{kbit} / \mathrm{s}$ and a PRR of

$$
p(S N R)=\left(1-1 / 2 e^{-\frac{S N R}{1.28}}\right)^{8 f},
$$

where $f$ is the frame size, i.e., the number of bytes in each packet. With a frame size of $f=50$, a MICA2 mote on the limit of losing contact, with $\mathrm{SNR}=5 \mathrm{~dB}$, would receive $1.8 \%$ of the packets and thus have an effective bandwidth of 340 bits/s. Sending a 10 kbyte camera image would then take 3 min 55 s. Gaining $5 \mathrm{~dB}$ would raise the bandwidth to 17.7 $\mathrm{kbit} / \mathrm{s}$, reducing the sending time to 4.5 seconds.

\section{CONCLUSION AND FUTURE WORK}

We have formulated the problem of positioning an autonomous robot to avoid the negative effect of multipath fading 

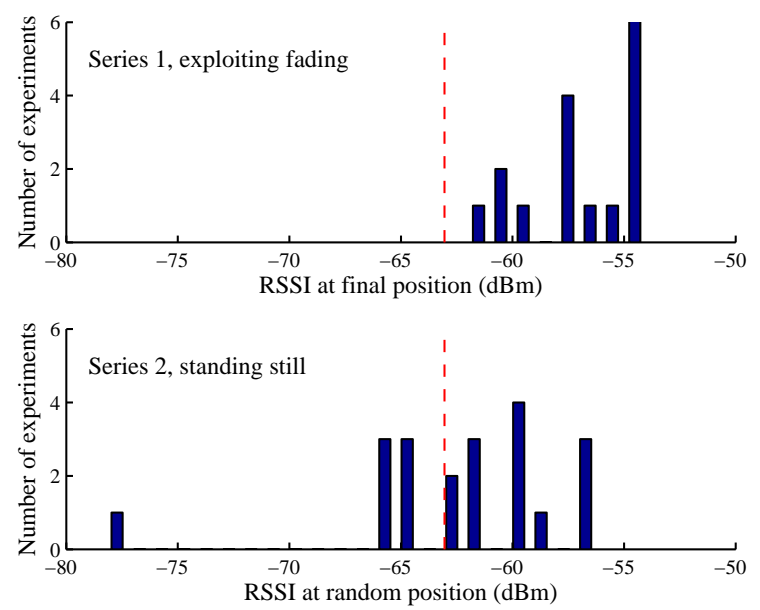

Fig. 17. Experiment results, measuring the RSS at the final position of the robot. The upper histogram shows the result when using the proposed approach, and the lower histogram shows what happened when the robot did not move from its (random) original position. The dashed line shows the local average RSS.

in radio communication. The problem is divided into two parts: first we provide an estimate of the number of points that it needs to sample to find a position that has a given signal strength. Then we suggest two possible sampling strategies to collect the required number of independent samples, without deviating too far from the original position.

This is a robust approach that works in several kinds of propagation environments and almost certainly avoids the deep fades where the signal can be attenuated as much as $20 \mathrm{~dB}$. In most cases it also gives a gain of $5 \mathrm{~dB}$ or more compared to the local mean signal power. This can be interpreted as using the unstructured environment as a directional antenna. The performance of this strategy was demonstrated in a simple experiment.

As mentioned in the introduction, the problem of multipath fading can also be countered in other ways, for example by antenna diversity as used in WLAN base stations. Our approach can be seen as a sort of antenna diversity over time, in case the robot is too small to host two antennas separated by at least half a wavelength. (Due to the asymmetry pointed out earlier, in some cases antenna diversity at the base station does not give the same advantages.) This also motivates moving groups of robots in hexagonal lattice formations with distance $\Delta$ between agents. Such a lattice represents the tightest possible formation offering antenna diversity gain for the group as a whole: ensuring that at least some of the robots have good signal strength.

In the future we would like to find criteria for how the variance of the RSS over time can be used to detect if the environment is no longer static.
Then the robot could adapt its strategy to increase the search space, exploit shadow fading instead or simply stop making adjustments to its position. Finally, we would like to measure the fading properties of other environments to further validate our approach.

\section{ACKNOWLEDGMENTS}

The authors would like to thank Carlo Fischione and Lucia Pallottino for useful discussions, and Simon Berg for building the measurement robot. This work was partially supported by the Swedish Defence Materiel Administration, the European Commission through the RUNES and HYCON projects, the Swedish Research Council, and the Swedish Foundation for Strategic Research.

\section{REFERENCES}

[1] R. Arkin and T. Balch. Line-of-sight constrained exploration for reactive multiagent robotic teams. 7th International Workshop on Advanced Motion Control, July 2002.

[2] K. E. Årzén, A. Bicchi, G. Dini, S. Hailes, K. H. Johansson, J. Lygeros, and A. Tzes. A component-based approach to the design of networked control systems. European Journal of Control, 2007. To appear.

[3] Z. Butler and D. Rus. Event-based motion control for mobile sensor networks. IEEE Pervasive Computing, 2(4), 2003.

[4] Chipcon AS. CC2420 Datasheet 1.4. www.chipcon.com, 2006.

[5] F. Darbari, I. McGregor, G. Whyte, R. W. Stewart, and I. Thayne. Channel estimation for short range wireless sensor network. In Proceedings of the 2nd IEE/Eurasip Conference on DSP Enabled Radio, 2005.

[6] A. Drenner, I. Burtz, B. Kratochvil, B. J. Nelson, N. Papanikolopoulos, and K. B. Yesin. Communication and mobility enhancements to the scout robot. In Proceeding of the IEEE/RSJ International Conference on Intelligent Robots and System, 2002.

[7] T. C. Hales. An overview of the Kepler conjecture. http://www.math.pitt.edu/ thales/kepler98/sphere0.ps, 1998.

[8] M. A. Hsieh, A. Cowley, V. Kumar, and C. J. Taylor. Towards the deployment of a mobile robot network with end-to-end performance guarantees. In Proceedings of the IEEE International Conference on Robotics and Automation, 2006.

[9] M. F. Mysorewala, D.O. Popa, V. Giordano, and F.L. Lewis. Deployment algorithms and in-door experimental vehicles for studying mobile wireless sensor networks. In Proceedings of the 7th ACIS International Conference on Software Engineering, Artificial Intelligence, Networking, and Parallel/Distributed Computing, 2006.

[10] H. G. Nguyen, N. Farrington, and N. Pezeshkian. Maintaining Communication Link for Tactical Ground Robots. In AUVSI Unmanned Systems North America, 2004.

[11] D. O. Popa and C. Helm. Robotic deployment of sensor networks using potential fields. In Proceedings of the IEEE International Conference on Robotics \& Automation, 2004.

[12] D. Puccinelli and M. Haenggi. Multipath fading in wireless sensor networks: Measurements and interpretation. In Proceedings of the International Wireless Communications and Mobile Computing Conference, 2006.

[13] Gordon L. Stüber. Principles of mobile communication. Kluwer academic publishers, 1996.

[14] J.D. Sweeney, R.A. Grupen, and P. Shenoy. Active QoS flow maintenance in controlled mobile networks. In Proceedings of the Fourth International Symposium on Robotics and Automation, 2004.

[15] A. Wagner and R. Arkin. Multi-robot communication-sensitive reconnaissance. In Proceedings of the IEEE International Conference on Robotics and Automation, 2004.

[16] M. Zuniga and B. Krishnamachari. Analyzing the transitional region in low power wireless links. In Proceedings of the First IEEE International Conference on Sensor and Ad hoc Communications and Networks, 2004. 